\title{
A Provider's Perspective: Current Issues in Providing and Funding Complementary Medical Care
}

\author{
G. T. Lewith \\ Hon. Senior Research Fellow/Hon. Consultant Physician, University of Southampton
}

\section{Introduction}

The provision of complementary and alternative medicine (CAM) has grown dramatically over the last decade. Eisenberg et al. [1] have clearly documented this in the USA, showing a doubling of expenditure on CAM products and consultations over a 7-year period during the 1990s from 132 billion USD per annum to 27 billion USD per annum in 1997. A similar situation exists in Australia [2], with continuing demand and interest within all medical specialities, particularly general practice [3]; and the situation in the UK also appears to be the same. Approximately $40 \%$ of UK general practitioners now provide some form of complementary medical practice [4], and expenditure on complementary medical consultations in one south coast English town would suggest that the UK spends much the same per capita as the USA on CAM [5]. The UK position in relation to CAM use is summarized in table 1.

The provider's perspective is therefore of an industry that appears to be growing at between 10 and $15 \%$ per annum, irrelevant of the variable economic climate that has occurred during the 1990s. While such growth may provide a solution to the overproduction of doctors in some EEC countries, it also raises a number of important issues:

- Why do people seek CAM? Is it because the therapies are intrinsically effective or are there other equally important social, emotional and spiritual reasons driving the growth of CAM?

- Is CAM being provided in an appropriate and ethical manner? It is very difficult in the context of a rapidly growing speciality such as CAM to benchmark and maintain quality. This is particularly relevant with respect to medical unemployment, managed care within conventional medicine (CM), and the associated fall in medical salaries throughout the Western world.

- If CAM is here to stay, how should it be delivered? What are the models we have available for CAM delivery in the UK and how can they be developed on a worldwide basis?

- Among the many claims of CAM practitioners is that CAM is cheap, acts in a preventative manner and therefore can be seen as a viable alternative to the management of chronic illness. An issue that bedevils all those who pay for the provision of health care is its cost: Is CAM really cheap and effective?

There is little doubt that providers of CAM who work both in the public and private sectors will come under increasing pressure to provide answers with respect to the quality of CAM practice, its effectiveness and relative efficacy as well as its cost-effectiveness in comparison to the technological and often expensive conventional medical treatments available.

\section{Why Do People Seek CAM?}

The first people to identify the extent of CAM practice in the UK were Fulder and Monro [8]. Since 1985 a substantial number of papers have been published which look at the attitudes of those using CAM to both CAM and health in general. An interesting picture emerges, which suggests that a significant proportion of those using CAM require a different approach to health care than those seeking CM. Finnigan $[9,10]$ noted in 1989 that over $50 \%$ of those attending a specialist complementary medical center were not necessarily simply seeking a solution to their medical problem but rather wished to take an active part in providing that solution as part of their management of their own health. Moore et al. [11] noted in 1984 that increasing numbers of patients were seeking CAM because it related more closely to their own intuitive understanding of their health needs. More recently, Astin [12] has made similar suggestions. It has been suggested that CAM may be effective simply because of the consultation process itself; Thomas [13] was the first to measure the real power of positive therapeutic intervention, and this has been a recurring theme within CAM over the last two decades [14]. The nature of a CAM consultation, particularly in homeopathy, demands both time and depth, and it is easy to see how this could be both egalitarian and, in itself, therapeutic [15]. Vincent and Furnham [16] have written extensively about the knowledge and beliefs of patients seeking CAM. It is very clear that those who move towards CAM have a far greater 'locus of control' over their own health. They take responsibility for their

\section{KARGER}

Fax +497614520714

(ㄷ) 2000 S. Karger GmbH, Freiburg

E-mail Information@Karger.de Accessible online at:

www.karger.com
George T. Lewith, MA DM FRCP MRCGP

Centre for the Study of Complementary Medicine

51 Bedford Place

Southampton SO1 2DG (UK) 
Table1. Changing attitudes to CAM

\section{Public}

- An estimated 4-5 million people a year in the UK go to complementary practitioners.

- Approximately 14-20\% of patients with chronic disease have consulted complementary practitioners [6]

- About $75 \%$ of the public support NHS access to CAM.

- Continuing public skepticism about drugs and side effects.

- Continuing media optimism and support for complementary therapies.

- Increased public wish to take more responsibility for health choices.

Professional

- Greater emphasis on consumer choice and patient care.

- BMA's positive attitude to CAM supports good practice in cooperation, education and research [7]

- GMC has clarified that doctors may delegate treatment to nonmedical complementary practitioners if satisfied of their competence and if the general practioner maintains overall clinical responsibility.

- A BMA GMSC (1991) survey showed that 20-30\% of UK general practioners would like to provide main forms of CAM through their practices.

- Rising levels of CAM medical research and outcome study publication in mainstream peer-reviewed journals.

- Recent establishment of General Osteopathic Council and General Chiropractic Council as statutory bodies to regulate these professions.

- Strongly expressed interest among some CAM practitioners in developing an NHS focus for their work

BMA = British Medical Association; $\mathrm{GMC}=$ General Medical Council GMSC $=$ General Medical Services Committee.

own health rather than expecting others to resolve their problems. There is no clear and distinct group of patients seeking CAM, but rather a population of individuals with a tendency for those seeking CAM to be more knowledgeable about health, more in control of their health and more desirous of an involved and egalitarian management process in relation to their specific problem [17-19]. Patients do not perceive $\mathrm{CM}$ as being more effective for major lifethreatening conditions but feel that CAM has much to offer in both acute self-limiting illnesses and chronic illness [20-22]. These perceptions have, of course, very little to do with hard evidence; all those practicing within the field of CAM base their clinical decisions largely on their experience, and not on evidence from case studies, outcomes research, or randomized controlled trials because in many instances such evidence does not exist. In spite of this, a significant proportion of the general public perceives CAM as both effective and relevant [16]. This therefore raises a number of important issues about the public perception and practice of both CAM and CM.

$\mathrm{CM}$ perceives itself as firmly based in science. It therefore tends to impose its own model of illness, often using Latin or Greek as a paralanguage for both the diagnosis and the description of the treatments employed. There is a tendency therefore, firmly based in Victorian medicine, for conventional doctors to use their knowledge and professional skills in a paternalistic and sometimes pedagogic manner. CAM, on the other hand, is frequently practiced by nonmedically qualified individuals who are less institutionalized and professionalized. As a consequence their approach to their patients is significantly more egalitarian and individualized and invokes medical models that are less didactic, more flexible, and more at one with the patient's own intuitive health perceptions. It may therefore be the approach used within CAM rather than its absolute effectiveness, which is what attracts and helps patients. It may even be that both treatments are equally clinically ineffective in the management of a particular chronic illness but that CAM provides the basis upon which the individual suffering from a particular illness is better able to cope. As a consequence, CAM may be perceived as both more attractive and more effective because of the process involved rather than the direct biochemical or physiological effects of the treatment. CAM may induce psychoneuroimmunological changes, and it is through these mechanisms that effective therapy may occur [23].

This therefore suggests that providers and purchasers of health care need to understand much more about the increasing popularity of CAM. What is this telling us about health care in general and the patient's needs in particular? There should only be one form of health care, effective evidence-based health care, yet $\mathrm{CM}$, in spite of its avowed science base, seems not to be providing for the needs of those seeking help. We understand too little about this phenomena and need to work with sociologists and health psychologists so that we can understand more.

\section{Models of Care}

The delivery mechanisms for CAM within the UK have been outlined previously [24], but it would be appropriate to expand on these observations with particular reference to Foundation for Integrated Medicine's report on delivery mechanisms [25].

It is clear that mechanisms of delivery for CAM differs in various Western nations. There are, for instance, a variety of state-based laws in the USA that license the practice of homeopathy, acupuncture, and chiropractic. These can, by and large, be carried out by nonmedically qualified practitioners on a state by state basis. In the UK there is a common law right to practice medicine, and currently CAM practitioners are going through a process of statutory regulation. The situation in Europe is more complex and involves a whole range of different regulations which allow the nonmedically qualified practitioner to exist in Germany while banning the practice of medicine by anybody other than an appropriately qualified medical individual in France and Belgium [26]. As a consequence, the delivery mechanisms throughout Europe are both varied and variable.

The main mechanism for delivering CAM is through individual practitioners working independently within their own practices on an outpatient basis (table 2). In the majority of instances, these individuals are not part of a team and may have, as a consequence, very variable levels of knowledge, skill and experience in diagnosis and in the therapies they practice. Furthermore, even though they may be medically qualified, their practice of CAM may be motivated by their need to maintain practice income rather than their skill and interest in the subject.

The second common area is CAM centers. These may sometimes be monotherapeutic (homeopathic hospital and/or centers for tra- 
Table 2. Models of care

\section{Family practice}

Within general practice

- Funded (state or private).

- Private (out of pocket).

- Team or independen.

- By interested general practioners / physio nurses within the practice.

Independent CAM practitioners

- Working as a group.

- Working independently with no quality control.

\section{CAM centers}

- Sometimes monotherapeutic (homeopathic hospitals).

- Necessity for inpatient care (Royal London Homoeopathic and Münchener Modell).

- Primarily outpatient care (Airdale - Environmental Medical Unit).

- Landlords or real integrated centers?

- Primary, secondary or tertiary referral centers?

Integrated hospital-based units

- Truly integrated (e.g. palliative care).

- Financial incentives to attract patients.

- Quality practice.

- Indemnity and insurance issues in relation to CAM practice.

ditional Chinese medicine), may involve both inpatient and outpatient care, and are more likely to encompass a team approach with some degree of benchmarking and quality control. However, some CAM centers, particularly in the UK, are simply run by landlords who collect various disparate CAM practitioners under one roof. The only communality between these practitioners is that they all pay rent to the same individual, and a team-based approach may or may not emerge from the propinquity of their clinical relationships. CAM centers may be isolated from the practice of CM and therefore may not represent a truly integrated or coherent approach to the delivery of medical care.

Integrated hospital units form the third major group of CAM provision. These are becoming increasingly popular, particularly in the USA. In the UK, integrated CAM provision has occurred mainly in the area of cancer, in particular palliative care. Hospital-based units may have financial incentives for providing CAM to attract patients into the private sector. They may also experience real problems in relation to the indemnity and insurance issues within CAM practice provided by both medical and nonmedically qualified individuals.

It is important to emphasize that good-quality medical care must be patient-centered as well as being integrated and evidencebased.

If we are going to provide CAM in response to patients' demand, then we must consider whether the provision of CAM is affordable, accessible, appropriate, acceptable, and efficient (table 3). CAM has little value if it is not fundamentally accessible and is open to great criticism if it is not used appropriately. We may, for instance, find CAM particularly effective in the context of palliative care, but CAM techniques are unlikely to be effective diagnostic and therapeutic tools for the initial management of malignancy. The integration and cooperation between those practicing CAM and conventional medicine are vitally important issues for us to ad-
Table 3. Is the provision of CAM appropriate?

\begin{tabular}{lll}
\hline & With cooperation & Without cooperation \\
\hline $\begin{array}{lll}\text { Patient } \\
\text { groups }\end{array}$ & $\begin{array}{l}\text { target patient groups e.g. } \\
- \text { chronic illness }\end{array}$ & $\begin{array}{l}\text { inappropriate referral } \\
\text { - magic? }\end{array}$ \\
& - high utilizers & \\
Outcome & appropriate treatment & all patients and cope with all \\
& protocols: managed care & illnesses with no inter- \\
& and referral back to CM & referral mechanisms \\
Structure & properly qualified CAM & independent practice possibly \\
& practitioners working in a & inappropriate and \\
& team with CM practitioners & dangerous treatment \\
& designed to meet patients & \\
& needs & \\
\hline
\end{tabular}

Table 4. The provision of CAM

\begin{tabular}{ll}
\hline With cooperation & Without cooperation \\
\hline Is it accessible? & - random \\
- location & - private practice \\
- affordable & - safe? \\
- timely & - no feedback \\
- team feedback & \\
Is it acceptable? & - private provision reflecting \\
- reflects health care needs & patient and practitioner needs \\
- patient satisfaction & \\
Is it effective? & \\
- maximum use of resources & \\
- team work & \\
- monitor outcome & \\
- evidence-based choices & \\
- efficient service with optimal treatment \\
\hline
\end{tabular}

dress, from the perspective of both the patient and the practitioner. Is the patient getting the most appropriate treatment? Are errors of diagnostic and therapeutic omission exposing the patient to real danger? If CAM is to continue to develop and grow as part of our health care provision, then it must do so on the basis of the provision of good quality and soundly evidence-based health care. This evidence cannot be solely based on randomized controlled trials, but those funding health care will only continue to do so if there is evidence supporting their financial decisions. We must consider clinical audit, the development of integrated clinical teams, and agreed best practice treatment regimes and subsequent benchmarking based on appropriate outcomes research as essential research tools within these clinical contexts (tables 3 and 4).

\section{The Effectiveness of Complementary Medicine}

There is no doubt that any clinical service provider wishes to make effective treatments available to their patients, a similar situation exists in both CAM and CM. The enthusiasm of the consumer has been the main driving force behind the expansion of CAM over the last decade, an enthusiasm driven by a variety of agendas some of which have already been discussed. Hard evidence in the con- 
text of randomized controlled trials and systematic reviews is limited within the field of CAM, partly by lack of research expertise [27], certainly by a lack of available funding for research [28], and very definitely by the limited capacity that exists to conduct highquality research within this field [27]. There are notable exceptions: For instance systematic reviews of the effect of acupuncture in nausea show definitive effects [29]. Linde et al. [30, 31] have been responsible for some excellent high-quality systematic reviews both in the field of herbal medicine [30] and within homeopathy [31]. These demonstrate that CAM is far from just being a question of magic.

In spite of the advances that have been made over the last decade, far more research is required both into the effects of individual interventions in specific diseases and into the effects of the commonly used packages of intervention that are frequently employed by complementary therapists (for instance the use of acupuncture, herbal medicine, diet and relaxation techniques which may be employed simultaneously in the treatment of headache or hypertension). Both providers and funders need to understand the subtle messages that patients are giving us about why they are seeking treatment and why the treatments may be effective. We have to work in partnership with our patients while at the same time giving them the freedom to pursue their own personal journey in relation to their own management of their illness. CM has tended not only to be paternalistic in terms of treatment provision, but also in terms of its established research agenda. CAM provides for us an opportunity to move away from that agenda and explore with patients what they perceive to be effective about the treatments that we can offer [3]. Evidence of effectiveness must involve an evidence-based hierarchy that includes case studies, clinical audit, outcomes research and randomized controlled trials [32].

\section{Cost-Effectiveness}

A number of claims have been made that suggest CAM is a cheap and effective mechanism for managing illness. Morgan and Brazier [33] have provided an excellent introduction to the principles of health economics in relation to CAM. As with much CAM research, there is little conclusive evidence to suggest that CAM is cheaper than conventional medicine. The case of homeopathy is perhaps a relevant example. Studies conducted by the Department of Social Security in France [33] suggest that total costs per physician associated with homeopathic care were half of that of conventional primary care physicians. However, this particular analysis does not clarify whether homeopathic physicians spent much longer with their patients than those attending for conventional care. It is clear that patients seeking homeopathic care are 32 times less likely to have certified sickness than those seeking conventional care. Unfortunately, we do not know whether these two populations are comparable both for their demography and their own personal attitudes to managing their health [34]. We are only too aware that different social groups react to illness in a different way and furthermore that attitudes to illness are an important governor

Current Issues in Providing and

Funding Complementary Medical Care of behavior, particularly in relation to time lost from work. It is, however, very clear that homeopathic remedies are much cheaper than conventional medication [34, 35]. Patients attending homeopathic physicians in both England and France spent far less on medication than they would had they visited conventional family physicians [34].

Information from both the Royal London Homoeopathic Hospital and the Glasgow Homoeopathic Hospital provides a very similar story. A large percentage of patients attending these institutions felt that effective health care had been delivered, and over $30 \%$ attending the Royal London Homoeopathic Hospital had stopped their conventional medications [36]. In Glasgow, of the patients attending the Homoeopathic Hospital $81 \%$ had a condition for which conventional medical treatment was not effective, and after 1 year $60 \%$ of these patients had experienced significant improvement with approximately $40 \%$ having substantially reduced their conventional medication while not requiring further hospital-based follow-up [32]. These figures are impressive but again deal with a small population of possibly self-referred patients requiring homeopathic treatment. In reality, this tells us very little about the effect that homeopathy might have on the population in general. Doubt must be thrown on the cost-effectiveness of CAM interventions by more generalized population studies such as that produced by Sommer et al. [37]. In their study 7,500 members of Switzerland's largest health insurer were offered free CAM cover for a period of 3 years. The clients were found to be using alternative medicine in addition to orthodox medical care, not instead of it. A small percentage of the experimental group took advantage of the free offer $(6.6 \%)$, and only $1 \%$ used CAM exclusively. The insurers could define no overall health improvement in the subjects using CAM. Therefore, the concept of CAM acting in a preventative fashion is questioned by this study. It appeared that CAM added to the insurers costs rather than minimizing them. Suggestions that homeopathy may be cost-effective in the context of dental practice [38] or that acupuncture may be of major cost-benefit in the treatment of severe angina [39] must all be treated with some degree of caution as the studies are simplistic and do not follow the fundamental principles of good health economics [33]. Further research is desperately needed in this area, and protocols such as those developed by Thomas et al [40], may provide us with information in the future about the cost-effectiveness of CAM interventions.

\section{Conclusion}

There is no doubt that we are seeing an explosion in the use of CAM throughout the Western world; but it is unclear exactly what this means. Is it a bid by patients to detach themselves from medical control or is it really because these therapies offer us new and effective approaches to the management of chronic intractable problems. Some individuals undoubtedly look at CAM as a magic solution to their illness, CAM has failed to live up to such unrealistic promise. It may perhaps be more realistically thought of as a mechanism for safely controlling and managing long-term illness 
with the minimum of side effects. As providers, we need to work with those funding CAM care in order to develop appropriate integrated treatment protocols that will allow patients to be provided with high-quality medical intervention, whether it is CAM or CM. Appropriate treatment without dogma and with good clinical governance must be at the core of our medical provision. Clearly there will be cost implications, and we must address the economic aspects of CAM with an openness and honesty that has so far been lacking. Many suggestions have been made that CAM offers a safe, cheap intervention. It may be safe, but when the therapist's time is taken into account, is it really cheap? Probably the most important issue that we need to come to grips with early in this decade is to benchmark what we consider to be ideal treatment protocols for particular conditions, bearing in mind of course the individualization and flexibility that CAM requires. Once we have achieved this, we can then begin to evaluate packages of treatment and employ the process of clinical audit and governance to benchmark quality. Patients have a right to the provision of good-quality medical care whether they seek complementary or conventional medicine. It is our duty as providers to look for the most effective and indeed the most cost-effective mechanisms for such provision.

\section{References}

1 Eisenberg DM, Davis RB, Ettner SL, Appel S, Wilkey S, Van Rompay M et al: Trends in alternative medicine use in the United States, 19901997. JAMA 1998;280:1569-1575.

2 MacLennan AH, Wilson DH, Taylor AW: Prevalence and cost of alternative medicine in Australia. Lancet 1996;347:569-573.

3 Lewith GT: Complementary and alternative medicine: An educational, attitudinal and research challenge. Med J Aust 2000;172:102-103.

4 Thomas K, Fall M, Parry G, Nicholl J: National survey of access to complementary health care via general practice. 1995. University of Sheffield. Ref Type: Report.

5 Dolan G, Lewith G: Complementary medicine outside the NHS: A pilot study in Southampton. J Altern Complement Med 1999;5:297-300.

6 RCCM: Public usage of complementary medicine: An overview. 1997. London, Research Council for Complementary Medicine Information Service. Ref Type: Report.

7 British Medical Association: Complementary Medicine: New Approaches to Good Practice. Oxford, Oxford University Press, 1993.

8 Fulder SJ, Munro RE: Complementary medicine in the UK, patients, practitioners and consultations. Lancet 1985;ii:542-545.

9 Finnigan MD: Complementary medicine: Attitudes and expectations, a scale for evaluation. Complement Med Res 1991;5:79-82.

10 Finnigan MD: The Centre for the Study of Complementary Medicine: An attempt to understand its popularity through psychological, demographic and operational criteria. Complement Med Res 1991;5:83-87.

11 Moore J, Phipps K, Marcer D, Lewith G: Why do people seek treatment by alternative medicine? BMJ 1985;290:28-29.

12 Astin JA: Why patients use alternative medicine? JAMA 1998;279:1548-1553.

13 Thomas KB: General practice consultations: Is there any point in being positive? BMJ 1987; 294:1200-1202.

14 Lewith GT: Reflections on the nature of the consultation. J Altern Complement Med 1998;4:321323.

15 Mitchell A, Cormack M: The Therapeutic Relationship in Complementary Health Care. Edinburgh, Churchill Livingstone, 1998.

16 Vincent C, Furnham A: Knowledge, Attitudes and Beliefs of Patients of Complementary Practitioners. Complementary Medicine. A Research
Perspective. Chichester, Wiley \& Sons, 1997, pp 97-117.

17 Furnham A, Vincent C, Wood R: The health beliefs and behaviours of three groups of complementary medicine and a general practice group of patients. J Altern Complement Med 1995; 1:347-359.

18 Furnham A, Bhagrath R: A comparison of health beliefs and behaviours of clients of orthodox and complementary medicine. Br J Clin Psychol 1993;32:237-246.

19 Furnham A, Kirkcaldy B: The medical beliefs and behaviours of orthodox and complementary medicine clients. Br J Clin Psychol 1996;35:49-62.

20 Vincent C, Furnham A, Willsmore M. The perceived efficacy of complementary and orthodox medicine in complementary and general practice patients. Health Education Research 1995; 10:395-405.

21 Vincent C, Furnham A: The perceived efficacy of complementary and orthodox medicine: Preliminary findings and the development of a questionnaire. Complement Ther Med 1994;2:128 134

22 Vincent C, Furnham A: The perceived efficacy of complementary and orthodox medicine. Complement Ther Med 1997;5:85-89.

23 Nixon P, King J: Ischemic heart disease: Homeostasis and the heart; in Watkins A (ed): MindBody Medicine. A Clinician's Guide to Psychoneuroimmunology. Edinburgh, Churchill Livingstone, 1997 pp 41-73.

24 Lewith GT, Wakins AD: Unconventional therapies in asthma: An overview. Allergy 1996; 51:761-769.

25 Peters D: Complementary and Alternative Medicine. Report of the Delivery Mechanisms Working Group. 1-27. 1997. London, Anne Wadsworth Associates. Ref Type: Report

26 Lewith G, Aldridge D (eds): Complementary Medicine and the European Community. Saffron Walden, C W Daniels, 1991.

27 Foundation for Integrated Medicine on behalf of the Steering Committee for the Prince of Wales Initiative on Integrated Medicine. Integrated healthcare. A way forward for the next five years? Discussion document. 1997. London, The Foundation for Integrated Medicine. Ref Type: Report.

28 Lewith G, Ernst E, Mills S, Fisher P, Monckton J, Reilly D, et al: Complementary medicine must be research led and evidence based. BMJ 2000; 320:188.
29 Vickers AJ: Can acupuncture have specific effects on health? A systematic review of acupuncture antiemesis trials. J R Soc Med 1996;89:303311.

30 Linde K, Ramirez G, Mulrow CD, Pauls A, Weidenhammer W, Melchart D: St John's Wort for depression - an overview and meta-analysis of randomised clinical trials. BMJ 1996:313:253-258.

31 Linde K, Clausius N, Ramirez G, Melchart D, Eitel F, Hedges LV, et al: Are the clinical effects of homoeopathy placebo effects? A meta-analysis of placebo-controlled trials. Lancet 1997;350: 834-843.

32 Lewith G, Reilly D: Integrating the complementary; in Dyer E (ed): NHS Year Book. London, Medical Information Systems, 1999, pp 46-48.

33 Morgan A, Brazier J: Economic evaluation of complementary therapies; in Lewith GT, Walach H, Jonas WB (eds): Clinical Research Methodology for the Complementary Therapies, Edinburgh, Churchill Livingstone, 2000, in press.

34 Ullman D: Homeopathy and managed care: Manageable or unmanageable. J Altern Complement Med 1999;5:65-73.

35 Swayne J: The cost and effectiveness of homoeopathy. Br Homoeopath J 1992;81:148-150.

36 Sharples F, van Haselen R: Patients perspective on using a complementary medicine approach to their health: A survey at the Royal London Homoeopathic Hospital NHS Trust. 1998. Ref Type: Report.

37 Sommer JH, Burge M, Theiss R: A randomized experiment of the effects of including alternative medicine in the mandatory benefit package of health insurance funds in Switzerland. Complement Ther Med 1999;7:54-61.

38 Feldhaus HW: Cost-effectiveness of homoeopathic treatment in a dental practice. Br Homoeopath J 1993;82:22-28.

39 Ballegaard S, Norrelund S: Cost-benefit of combined use of acupuncture, shiatsu and lifestyle adjustment for treatment of patients with severe angina pectoris. Acupunct Electrother Res 1996; 21:187-197.

40 Thomas KJ, Fitter M, Brazier J, MacPherson H, Campbell M, Nicholl JP, et al: Longer term clinical and economic benefits of offering acupuncture to patients with chronic low back pain assessed as suitable for primary care management. Complement Ther Med 1999;7:91-100. 